JOSING: Journal of Nursing and Health

Volume 1, Nomor 2, Juni 2021

e-ISSN: 2745-7877

p-ISSN: 2746-0851

DOI: https://doi.org/10.31539/josing.v1i2.2122

\title{
DISCHARGE EDUCATION FOR MILD TRAUMATIC BRAIN INJURY PATIENTS AT EMERGENCY DEPARTMENT
}

\author{
Praneed Songwathana ${ }^{1}$, Nurhayati ${ }^{2}$ \\ Prince of Songkla University ${ }^{1}$ \\ University of Muhammadiyah Bengkulu ${ }^{2}$ \\ nurhayati@umb.ac.id ${ }^{2}$
}

\begin{abstract}
This study aims to review the literature on educational interventions for the discharge of mild traumatic brain injury (mTBI) patients in the Emergency Room (IGD). Database searches were performed based on PICO queries and keywords. using ProQuest, ScienceDirect, Scopus, and PubMed for the period 2012-2019. Ten studies were reviewed. The findings revealed the contents of discharge information for mTBI patients including diagnosis, signs and symptoms, treatment and medication, pain management, home care, and ED return instructions. Four methods of discharge intervention are commonly used in the ED; written, spoken, animated and moving videos, and printed instructions measured over two time periods, either pre-discharge in the ED or followup 1-2 weeks post-discharge at home. In conclusion, combined teaching was more effective in terms of level of knowledge, understanding, and ED repeat visits.
\end{abstract}

Keywords: Discharge education, Emergency, Minor Traumatic Brain Injury

\section{INTRODUCTION}

Traumatic Brain Injury (TBI) is the rapidly increasing outcome of road traffic accident. The World Health Organization (WHO) estimated that TBI will be a leading cause of death and disability by 2020 with approximately 10 million people affected each year (Chaboyer et al, 2013). For that TBI affected severity can range from mild to severe $^{2}$. However, Kempe study (2014) found that Mild Traumatic Brain Injury (mTBI) compose from 80 to 90 percent of citizen hospital admissions accounts for approximately 75 percent of those injured.

The emergency department (ED) is the initial point of contact for TBI management. Research by Minen et al., (2016) explained that the rate of emergency department visits for TBI has been rising. With the rapid growth of the ED visits, the ED return rates can be considerable. Revisits to the ED have been put forward as markers of quality as they are influenced by diagnostic accuracy, education at the time of discharge, and effective care on the first visit. Revisit after discharge high with a serious complication. Research by Lane et al., (2017) explained that after a mTBI occurs, there is a temporal window of brain vulnerability that makes it more susceptible to further injury. During this window, the complication might happen such as diffuse cerebral swelling as well as a chronic postconcussion syndrome and long-term cognitive, psychiatric, and neurobehavioral problems. To prevent that complication and revisit, the adequacy and comprehensive of discharge education are very important for transfer patient to the home. 
Discharge education at emergency department still become a crucial problem. The previous study explained that 78 percent of patients discharged from an ED did not understand regarding homecare instructions and 20 percent were lack of apprehension (Marshall et al, 2012). The barrier of discharge education at ED include limited of time, there is no instruction standard form, lack of attention from the patient, a limited resources, crowded of patients and environment, and lack of nurses continuity (Bay \& Strong, 2011).

Mild Traumatic Brain Injury (mTBI) may not have signs and symptoms of intracranial haemorrhage in the emergency department (ED). The nurse needs to educate patients before discharge from ED to the home to improve quality of care of the patient. The nurse needs to identify which intervention to be adopted in the limited time that incorporates delivery of ED. Evidence-based clinical practice guideline in discharge education can simplify an appropriate management and evaluation of mTBI patient. The previous studies related to discharge education showed the adequacy of discharge education was influenced the successes in reducing readmission, ED revisit, and improved patient safety (Ismail et al, 2016). However, there is a lack of literature which is illuminated or synthesized the discharge education intervention to determine the most effective strategies to ensure patient comprehension of discharge education from ED. This study aims to review the evidence-based of discharge education intervention for mTBI patient at the emergency department.

\section{RESEARCH METHODS}

Databases searched was conducted based on PICO questions (Population, Intervention, Comparison, and Outcome); What is the most effective discharge education for mTBI patient at ED; mTBI patient (P), Emergency discharge instructions or education (I), Teaching methods (C), Retention of knowledge, Patient's complication and ED revisit $(\mathrm{O})$ by ProQuest, ScienceDirect, Scopus, and PubMed in period time 2012-2019. There were 280 articles that filtered out where 119 articles potentially relevant for abstract review, 54 reports for full text review, and 10 articles included for representing review. All of the relevant articles were done by using Joanna Briggs Institute's tool.

\section{RESULTS \\ Content of Discharge Education \\ Diagnosis}

Teaching early in the course of mild traumatic brain injury (mTBI) often focuses on reinforcing information given to the caregiver about patien condition and prognosis.

\section{Sign and Symptom}

This information may focus on interpretation of changes in the patients' physical and physchological responses that may occur in post-acute period of injury. The most common sign and symptoms of mTBI patient include confusion, disorientation, impaired consciousness, seizures, headache, dizziness, irritability or emotional lability, fatigue, poor concentration, memory difficulties, and intelectual dysfunction that may occur from 1 week to 1 year after the initial injury. Hypertermia may indicate brain stem damage, educted the caregiver to maintain patients' body temperature less than $38^{\circ} \mathrm{C}$ by applying cool-bath, placement of ice packs, or air-filled cooling devices. 


\section{Medication and Treatment}

Medication information includes indication, dosage, side effects, and how to manage the side effects at home. Antibiotic may be prescribed especially patient with an open head injury to prevent infection. Antiseizure medication or anticonvulsan may be given to reduce the risk of seizures activity that significantly increased methabolic, cerebral blood flow, and Intracranial Pressure (ICP).

\section{Pain Management}

Provide information about appropriate pain management by using various type of analgesia and weaning from pain medication. Understanding all analgesia options help the client in decision making for pain relief. Combination of pharmacological and nonpharmachological theraphy is adequate to reduce pain such as placement of ice packs therapy.

\section{Home Care}

Home care is the important thing that should be given to the patient and caregiver before discharge. This information include self-care ability, wound care for an open head injury, how to prevent complication, timing for a follow-up appoinment with the appropriate pysician, and how to connect with health services nearest to them.

\section{Return ED Instruction}

Clinical warning sign information that should trigger patient and caregiver to return immediately to ED. Educate the patient and caregiver to notify the physician or bring the patient to the emergency department if they occur; difficulty in awakening or speaking, confusion, severe headache, vomiting, and weakness of one side of the body to get an emergency care.

Table. 1

Comparison of Information Contents

\begin{tabular}{lccccc}
\hline \multirow{2}{*}{ Citations } & \multicolumn{5}{c}{ Information Content of Discharge Education } \\
\cline { 2 - 7 } & Diagnosis & $\begin{array}{c}\text { Sign and } \\
\text { Symptoms }\end{array}$ & $\begin{array}{c}\text { Medication } \\
\text { and } \\
\text { Treatment }\end{array}$ & $\begin{array}{c}\text { Home } \\
\text { Care }\end{array}$ & $\begin{array}{c}\text { Return ED } \\
\text { Instruction }\end{array}$ \\
\hline Ismail et al (2015) & $\sqrt{ }$ & $\sqrt{ }$ & $\sqrt{ }$ & $\sqrt{ }$ & - \\
Engel et al (2012) & $\sqrt{ }$ & $\sqrt{ }$ & $\sqrt{ }$ & $\sqrt{ }$ & $\sqrt{ }$ \\
Zavala et al (2011) & $\sqrt{ }$ & $\sqrt{ }$ & $\sqrt{ }$ & - & - \\
Lane et al (2017) & - & - & - & - & - \\
Bay \& Strong (2011) & $\sqrt{ }$ & $\sqrt{ }$ & $\sqrt{ }$ & $\sqrt{ }$ & - \\
Currant et al (2016) & - & $\sqrt{ }$ & & $\sqrt{ }$ & - \\
Kempe et al (2014) & - & $\sqrt{ }$ & $\sqrt{ }$ & $\sqrt{ }$ \\
Qureshi et al (2013) & - & $\sqrt{ }$ & - & - & - \\
Kornburger et al (2013) & - & - & - & - & $\sqrt{ }$ \\
Buckley et al (2013) & - & - & - & $\sqrt{ }$ & - \\
\hline
\end{tabular}

\section{Methods of Discharge Education \\ Written Instructions}

Written instruction is the most common discharge education methods in emergency setting. This method is handwritten by physicians and nurses that includes 
diagnosis, treatment, self-care, and follow up an appointment. The benefit of this method is a simple instruction that is specific to the patient condition.

\section{Verbal or Teach-Back Instructions}

Verbal or teach back instruction is also used in the emergency setting. Nurses teach the patient and caregiver about the concept of mTBI by oral presentation. Then patient repeats or demonstrates those instructions using their own words.

\section{Animation and Moving Video Instructions}

Nurses provide animation and moving video with mTBI content such as diagnosis, treatment, disease process, and discharge instruction at home. The patient watches the video after receiving their usual care. This method is feasible for all of the patient and caregiver, particularly the patient who has low education, they can understand easily using this method.

\section{Printed Instructions}

This method has a more attractive look as it uses a combination of words and pictures for the delivery of information such as a brochure, leaflet, booklet, and discharge instruction sheets. The patient can take those sheets everywhere and re-read as much as they want.

Table. 2

Comparison of Education Methods

\begin{tabular}{lcccc}
\hline \multirow{2}{*}{ Citations } & \multicolumn{4}{c}{ Methods of Discharge Education } \\
\cline { 2 - 5 } & $\begin{array}{c}\text { Verbal } \\
\text { Instructions }\end{array}$ & $\begin{array}{c}\text { Written } \\
\text { Instructions }\end{array}$ & $\begin{array}{c}\text { Animation } \\
\text { and Moving } \\
\text { Video }\end{array}$ & $\begin{array}{c}\text { Printed } \\
\text { Instructions }\end{array}$ \\
\hline Ismail et al (2015) & $\sqrt{ }$ & $\sqrt{ }$ & $\sqrt{ }$ & - \\
Engel et al (2012) & - & $\sqrt{ }$ & - & $\sqrt{ }$ \\
Zavala et al (2011) & - & $\sqrt{ }$ & - & - \\
Lane et al (2017) & - & $\sqrt{ }$ & - & $\sqrt{ }$ \\
Bay \& Strong (2011) & - & $\sqrt{ }$ & $\sqrt{ }$ & $\sqrt{ }$ \\
Currant et al (2016) & $\sqrt{ }$ & - & - & $\sqrt{ }$ \\
Kempe et al (2014) & - & $\sqrt{ }$ & - & $\sqrt{ }$ \\
Qureshi et al (2013) & - & - & $\sqrt{ }$ & - \\
Kornburger et al (2013) & $\sqrt{ }$ & $\sqrt{ }$ & - & $\sqrt{ }$ \\
Buckley et al (2013) & $\sqrt{ }$ & &
\end{tabular}

\section{Factors associated with the quality of discharge education \\ Content of Information}

The content of discharge information is the important thing to achieve the goal of discharge education. This content includes diagnosis, medication and treatment, sign and symptoms, home care instructions, pain management, and the urgency condition that patient needs to return to the emergency department immediately to avoid the worse complication.

\section{Delivery Methods}

The way of delivery information is very important in achieving successful information. for instance, the delivery methods may not be appropriate for the patient's 
condition. The patient who has a low level of education may not be able to read of written or printed information. The information may be delivered too quickly due to lack of time and without considering each patient learning needs.

\section{Implementation of Discharge Education}

Implementation of information after discharge at home by the patient and caregiver often becomes a new problem for them. The patient may not be able to apply discharge information at home due to lack of confidence and poor experience about their case.

\section{DISCUSSION}

\section{Content of Discharge Education \\ Diagnosis}

Teaching early in the course of mild traumatic brain injury (mTBI) often focuses on reinforcing information given to the caregiver about patien condition and prognosis (Smeltzer et al, 2010). Giving patient and caregiver the truthful information about their diagnosis will help them to become informed participants in health care decision (Bay \& Strong, 2011; Engel et al., 2012; Ismail et al., 2015; Zavala \& Shaffer, 2011).

\section{Sign and Symptom}

This information may focus on interpretation of changes in the patients' physical and physchological responses that may occur in post-acute period of injury. The most common sign and symptoms of mTBI patient include confusion, disorientation, impaired consciousness, seizures, headache, dizziness, irritability or emotional lability, fatigue, poor concentration, memory difficulties, and intelectual dysfunction that may occur from 1 week to 1 year after the initial injury (Smeltzer et al, 2010). Hypertermia may indicate brain stem damage, educted the caregiver to maintain patients' body temperature less than $38^{\circ} \mathrm{C}$ by applying cool-bath, placement of ice packs, or air-filled cooling devices.

Inform the patient that mTBI patient increased an risk of postraumatic seizures in immediate (within 24 hours after injury), early (within 1 to 7 days after injury), or late period (more than 7 days after injury). If the patients have open head injury, informed them about wound infection signs such as redness, swelling, or foul drainage (Bay \& Strong, 2011; Curran et al., 2016; Engel et al., 2012; Ismail et al., 2015; Kornburger et al., 2013; Qureshi et al., 2013; Zavala et al., 2011).

\section{Medication and Treatment}

Medication information includes indication, dosage, side effects, and how to manage the side effects at home. Antibiotic may be prescribed especially patient with an open head injury to prevent infection. Antiseizure medication or anticonvulsan may be given to reduce the risk of seizures activity that significantly increased methabolic, cerebral blood flow, and Intracranial Pressure (ICP). Antipyretic is used to decrease body temperature and metabolic need of brain, thereby reducing the volume of blood to the brain to supply oxygen and glucose (Bay \& Strong, 2011; Engel et al., 2012; Ismail et al., 2015; Kempe et al., 2014; White et al, 2013; Zavala et al., 2011). 


\section{Pain Management}

Provide information about appropriate pain management by using various type of analgesia and weaning from pain medication. Understanding all analgesia options help the client in decision making for pain relief. Combination of pharmacological and nonpharmachological theraphy is adequate to reduce pain such as placement of ice packs therapy. beside that, educate the patient how to report pain through verbally or facial expression is also important in pain management (Nurhayati et al., 2019; Smeltzer et al., 2010).

\section{Home Care}

Home care is the important thing that should be given to the patient and caregiver before discharge. This information include self-care ability, wound care for an open head injury, how to prevent complication, timing for a follow-up appoinment with the appropriate pysician, and how to connect with health services nearest to them. Furthermore, encourage the patient to perform range-of-motion exercises to prevent deep vein thrombosis, encourage them as much activity as possible (Bay \& Strong, 2011; Curran et al., 2017; Engel et al., 2012; Kempe et al., 2014; Ismail et al,. 2015).

\section{Return ED Instruction}

Clinical warning sign information that should trigger patient and caregiver to return immediately to ED. Educate the patient and caregiver to notify the physician or bring the patient to the emergency department if they occur; difficulty in awakening or speaking, confusion, severe headache, vomiting, and weakness of one side of the body to get an emergency care (Engel et al., 2012; Kempe et al., 2014; Kornburger et al., 2013).

\section{Methods of Discharge Education Written Instructions}

Written instruction is the most common discharge education methods in emergency setting. This method is handwritten by physicians and nurses that includes diagnosis, treatment, self-care, and follow up an appointment. The benefit of this method is a simple instruction that is specific to the patient condition. However, this method is not appropriate for the patient and caregiver who are not be able to read (Bay \& Strong, 2011; Buckley et al, 2013; Engel et al, 2012; Ismail et al, 2015; Kempe et al, 2014; Kornburger et al, 2013; Nurhayati \& Madsiri, 2019; Zavala et al, 2011).

\section{Verbal or Teach-Back Instructions}

Verbal or teach back instruction is also used in the emergency setting. Nurses teach the patient and caregiver about the concept of mTBI by oral presentation. Then patient repeats or demonstrates those instructions using their own words. If the patient gives incorrect feedback about the concept of injury, the nurse can clarify the information using another scripted sentence that is easy for the patient to understand. However, if the patients have memory impairment, they will difficult to recall and reexplain the information through verbal method (Buckley et al., 2013; Curran et al., 2016; Ismail et al., 2015; Kornburger et al., 2013). 


\section{Animation and Moving Video Instructions}

Nurses provide animation and moving video with mTBI content such as diagnosis, treatment, disease process, and discharge instruction at home. The patient watches the video after receiving their usual care. This method is feasible for all of the patient and caregiver, particularly the patient who has low education, they can understand easily using this method. Furthermore, it also has benefits for the nurse, in crowded condition with limited time of emergency care, the nurse is able to look after another patient while the mTBI patient watches the information video. The weakness of this method is the patient cannot ask the question directly if they are missing some part during watching the video (Bay \& Strong, 2011; Ismail et al., 2015; Kornburger et al., 2013).

\section{Printed Instructions}

This method has a more attractive look as it uses a combination of words and pictures for the delivery of information such as a brochure, leaflet, booklet, and discharge instruction sheets. The patient can take those sheets everywhere and re-read as much as they want. However, like written instruction, this method is not appropriate for the patients and caregiver who are not able to read (Bay \& Strong, 2011; Buckley et al., 2013; Engel et al., 2012; Kempe et al., 2014; Lane et al., 2017).

\section{Factors associated with the quality of discharge education Content of Information}

The content of discharge information is the important thing to achieve the goal of discharge education. This content includes diagnosis, medication and treatment, sign and symptoms, home care instructions, pain management, and the urgency condition that patient needs to return to the emergency department immediately to avoid the worse complication. There are some factors that influence the level of patient's understanding such as complicated content (the patients might be not familiar with medical words), the content may be too general to be meaningful and may not be tailored to patient needs.

\section{Delivery Methods}

The way of delivery information is very important in achieving successful information. for instance, the delivery methods may not be appropriate for the patient's condition. The patient who has a low level of education may not be able to read of written or printed information. The information may be delivered too quickly due to lack of time and without considering each patient learning needs.

\section{Implementation of Discharge Education}

Implementation of information after discharge at home by the patient and caregiver often becomes a new problem for them. The patient may not be able to apply discharge information at home due to lack of confidence and poor experience about their case.

Based on synthesizing of the articles, the contents of discharge information for mTBI patient include diagnosis, signs and symptoms, medication and treatment, pain managemnent, home care, and Instructions about ED revisit. Four discharge intervention methods were used at ED, which are written, verbal, animation and moving video, and printed instructions. The most common method of discharge education is written instruction. However, it is recommended to use more than 1 teaching method at 
the emergency department in order to develop good result in increasing the level of knowledge, the level of understanding, and to decrease mTBI patient revisit at ED. The information content should not be complicated, use common words, and be more specific to each patient's need. Delivery methods should be feasible for the patient condition. Health care provider nearby the patients' home is recommended to observe the implementation process by patient and caregiver.

Moreover, outcomes are evaluated twice in some studies to ensure the level of understanding and ability to take care after discharge as a follow-up. For example, 4 articles only evaluate the intervention in pre-discharge time in the emergency department by using questionnaire and checklist. Then 6 articles evaluate the intervention that they provide both in the pre-discharge and after-discharge period at home by using telephone call. However, most of the articles are descriptive study, they observed the discharge process and evaluate them, so it might be low evidence. Only few study that makes a comparison between usual care and another discharge methods like animation and moving video as an experimental group. The outcomes of discharge education were measured over two periods; either pre-discharge at ED or 1-2 weeks follow up post-discharge to ensure the level of understanding and self-care ability of the patient after returning home.

The quality of discharge education may be good depending on various factors including the content of information, delivery methods, and implementation by the patient and caregiver itself. The content of information should be not complicated, use common words that easy to understand by the patient, and more specifically to the patient problem. Besides that, for delivery methods should be feasible with patient condition, for instance, the nurse might not give written instruction for the patient who is not able to read. The health care provider nearby the patients' home should observe the patient regularly to achieve the good result in implementating of discharge information at home.

\section{CONCLUSION}

In this review, current knowledge regarding discharge education at the emergency department for mild traumatic brain injury is almost descriptive studies. Therefore, future investigations of discharge education need more studies intervention such as randomized control trials and experimental study to compare the effectiveness of each instruction. Then to improve the quality of care of the patient, the adequacy and comprehensive of discharge education is very important for transfer patient to the home. So the nurse needs to measure the patient readiness for discharge for further study.

\section{ACKNOWLEDGMENT}

The authors acknowledge the Higher Education Research Promotion and the Thailands' Education hub for Southern Region of ASEAN Countries Project Office of the Higher Education Comission for the financial support of this study. 


\section{REFERENCES}

Bay, E., \& Strong, C. (2011). Mild Traumatic Brain Injury: A Midwest Survey of Discharge Teaching Practices of Emergency Department Nurses. Advanced Emergency Nursing Journal, 33(2), 181-192.

Buckley, B. A., McCarthy, D. M., Forth, V. E., Tanabe, P., Schmidt, M. J., Adams, J. G., \& Engel, K. G. (2013) Patient Input Into the Development and Enhancement of ED Discharge Instructions: A Focus Group Study. Journal of Emergency Nursing, 39(6), 553-561. DOI: 10.1016/j.jen.2011.12.018

Chaboyer W, Ringdal M, Aitken L, Kendall E. (2013). Self-Care after Traumatic Injury and the Use of the Therapeutic Self-Care Scale in Trauma Populations. Journal of advanced nursing, 69(2), 286-294. DOI: 10.1111/j.1365-2648.2012.06005.X

Curran, J. A., Murphy, A., Burns, E., Plint, A., Taljaard, M., MacPhee, S., \& Bourque, M. (2017). Essential Content for Discharge Instructions in Pediatric Emergency Care: A Delphi Study. Pediatric Emergency Care, 34(5), 339-343. DOI: 10.1097/PEC.0000000000000986

Engel, K. G., Buckley, B. A., Forth, V. E., McCarthy, D. M., Ellison, E. P., Schmidt, M. J., \& Adams, J. G. (2012). Patient Understanding of Emergency Department Discharge Instructions: Where are Knowledge Deficits Greatest?. Academic Emergency Medicine, 19(9), E1035-E1044. http://ovidsp.ovid.com/ovidweb.cgi?

Ismail, S., McIntosh, M., Kalynych, C., Joseph, M., Wylie, T., Butterfield, R., \& Osian, S. R. (2016). Impact of Video Discharge Instructions for Fever and Closed Head Injury from the Emergency Department. The Journal of Emergency Medicine, 50(3), e177-e183. DOI: 10.1016/j.jemermed.2015.10.006

Kempe, C. B., Sullivan, K. A., \& Edmed, S. L. (2014). A Critical Evaluation of Written Discharge Advice for People with Mild Traumatic Brain Injury: What Should We Be Looking For?. Brain Injury, 28(12), 1551-1558.

Kornburger, C., Gibson, C., Sadowski, S., Maletta, K., \& Klingbeil, C. (2013). Using "Teach-Back" to Promote a Safe Transition from Hospital to Home: An Evidence-Based Approach to Improving the Discharge Process. Journal of Pediatric Nursing, 28(3), 282-291. DOI: 10.1016/j.pedn.2012.10.007

Lane, A. D., Berkman, M. R., Verbunker, D., Shekell, T., Bouska, M., Barnett, L. \&, Waterbrook, A. L. (2017). Retrospective Chart Analysis of Concussion Discharge Instructions in the Emergency Department. The Journal of Emergency Medicine, 52(2), 690-698. DOI: 10.1016/j.jemermed.2016.12.017

Marshall, S., Bayley, M., McCullagh, S., Velikonja, D., \& Berrigan, L. (2012). Clinical Practice Guidelines for Mild Traumatic Brain Injury and Persistent Symptoms. Canadian Family Physician, 58(3), 257-267. http://www.pubmedcentral.nih.gov/

Minen, M., Shome, A., Femia, R,, Balcer, L., Grudzen, C., \& Gavin, N. P. (2017). Emergency Department Concussion Revisits: Chart Review of the Evaluation and Discharge Plans of Post-Traumatic Headache Patients. The American journal of emergency medicine, 35(2), 365-367. DOI: 10.1016/j.ajem.2016.10.076

Nurhayati, N., \& Madsiri, M. (2019). Managing Acute Pain after Abdominal Surgery: Examples from Practice. Gastrointestinal Nursing, 17(8), 20-25. DOI: 10.12968/jprp.2020.2.2.78

Nurhayati, N., Praneed, S., \& Ratjai, V. (2019). The Quality of Discharge Teaching Perceived by Surgical Nurses Working in Public Hospitals of Indonesia. International Journal of Caring Sciences, 12(1), 100-106. http://search.ebscohost.com/login.aspx?direct=true\&db 
Qureshi, K. L., Hawley, C. A., Peachey, T., Cooke, M. W., \& Unsworth, L. (2013). Minor Head Injury in Children: Evaluating Written Discharge Advice Provided by Scottish Emergency Departments. Brain Injury, 27(13-14), 1549-1554. DOI: 10.3109/02699052.2013.837197

Smeltzer, S. C., Bare, B. G., Hinkle, J. L., \& Cheever, K. H. (2010). Brunner \& Suddarths' Texbook of Medical-Surgical Nursing. 12th ed. China: Lippincott William \& Wilkins

White, L., Duncan, G., \& Baumle, W. (2013). Medical-Surgical Nursing an Integrated Approach. 3rd ed. Canada: Delmar Cengage Learning

Zavala, S., \& Shaffer C. (2011). Do Patients Understand Discharge Instructions?. Journal of Emergency Nursing, 37(2), 138-140. DOI: 10.1016/j.jen.2009.11.008 